\title{
OBESIDADE ABDOMINAL E GRAU DE PROCESSAMENTO DOS ALIMENTOS EM MULHERES NA MENOPAUSA: ESTUDO RETROSPROSPECTIVO
}

Damiana Canali; Universidade de Caxias do sul; dcanali@ucs.br Carolina Pagnoncelli Gabrielli; Universidade de Caxias do Sul; carolinapgabrielli@gmail.com; Simone Bonatto; Universidade de Caxias do Sul; sbonatto1@ucs.br; Josiane Siviero; Universidade de Caxias do Sul; jsiviero@ucs.br

\section{RESUMO}

Objetivo: Verificar a associação entre obesidade abdominal e grau de processamento dos alimentos nas mulheres na menopausa. Métodos: Estudo transversal retrospectivo com mulheres na menopausa participantes de um programa de extensão da Universidade de Caxias do Sul. As variáveis analisadas foram: idade, estado civil, renda, escolaridade, idade da menopausa, se fez uso de reposição hormonal, Idade da menarca, número de filhos, se amamentou e horas de sono. Em relação às práticas alimentares observou-se o consumo de frituras, de líquidos e os alimentos conforme o grau de processamento. Resultados: Participaram do estudo 193 mulheres a maioria possuía idade $\geq 60$ anos $(67,4 \%)$. A prevalência de obesidade abdominal foi 76,2\% (IC 95\%, 70,1-82,2). Nas análises de associação com as características da amostra, encontrou-se associação significativa com a escolaridade $(p=0,05)$. Ao relacionar o consumo alimentar das mulheres na menopausa de acordo com o grau de processamento dos alimentos, verificou-se que as que consomem uma mediana maior de alimentos in natura e/ou minimamente processados não possuíam obesidade abdominal $(p=0,001)$ e não fizeram reposição hormonal $(p=0,04)$. Conclusão: A associação entre a obesidade abdominal e o grau de processamento dos alimentos se deu positivamente para mulheres na menopausa que consomem mais alimentos in natura e minimamente processados como um fator protetor para doenças crônicas não transmissíveis. Contudo, mais estudos relacionando as práticas e hábitos alimentares são essenciais, para uma fortalecer a atenção à saúde com a promoção e prevenção em todas as fases da vida.

Palavras-chave: Menopausa; Obesidade Abdominal; Mulheres; Alimentos Processados. 